\section{References}

${ }^{1}$ C. L. Smith, “Fuel Particle Behavior Under Thermal and Transient Conditions," ERDA Tech. Rept. No. GA-A12971, Oct, I, 1974.

${ }^{2}$ A. S. Shenoy and D. W. McEachem, "HTGR Core Thermal Design Methods and Analysis," ERDA Tech. Rept. No. GA-A12985, Dec. 31, 1974.

"'HTGR Accident !nitiation and Progression Analysis Status Report." ERDA Tech. Rept. No. GA-AI3617, Vols. I, II, and III, Jan. 1976.
'C. L. Smith, "SiC-Fission Product Reactions in HTGR TRISO UC $\mathrm{U}_{2}$ and UC, $\mathrm{O}_{2}$ Fissile Fuel: I"; this issue. pp. 600-606

${ }^{5}$ F. J. Homan, T. B. Lindemer, E. L. Long, T. N. Tiegs, and K. L. Beatty, "Stoichiometric Eftects on Pertormance of HTGR Fuels trom the U-C-O System,"

Nucl. Teclinol., 35 [2] 428-4l (1977).

BD P Harmon and C B Scott "Properties Influencing HTGR Coated Fuel Particle Performance," ibid. pp. 343-52.

${ }^{7}$ B. Myers. General Atomic Co,: private communication

\title{
Solubility Limits in Binary (Ca,Mn) Chalcogenides
}

\section{CHI-HUNG LEUNG}

\author{
Gibson Electric Inc., Delmont, Pennsylvania 15626
}

\section{LAWRENCE H. VAN VLACK ${ }^{*}$}

Department of Materials and Metallurgical Engineering, The University of Michigan, Ann Arbor, Michigan 48109

Solid solubilities progressively decrease in the systems CaSMnS, CaSe-MnSe, and CaTe-MnTe. In CaS-MnS, full solid miscibility extends from $1150^{\circ}$ to $1500^{\circ} \mathrm{C}$, the liquidus minimum. With CaSe-MnSe, this range is $1070^{\circ}$ to $1340^{\circ} \mathrm{C}$. The solid miscibility gap between $\mathrm{CaTe}$ and cubic $\mathrm{MnTe}$ intercepts the liquidus as a eutectic at $1050^{\circ} \mathrm{C}$. The solid solutions follow Vegard's law closely.

\section{Introduction}

$\mathbf{T}$ HE calcium and manganese chalcogenides offer an opportunity to examine the solubility limits in simple isomorphous systems. The compounds $\mathrm{CaS}, \mathrm{CaSe}, \mathrm{CaTe}, \mathrm{MnS}$, and MnSe possess the $\mathrm{NaCl}$ structure at all temperatures; $\mathrm{MnTe}$, which is hexagonal (NiAs structure) at $<1040^{\circ} \mathrm{C}$, has the $\mathrm{NaCl}$ structure at high temperatures. The present work is a report on the solubility limits in the binary systems CaS-MnS, CaSe-MnSe, and CaTe-MnTe, which involve cation substitution within each system.

\section{Previous Work}

The Group VI-A compounds of iron and manganese have been studied $^{1-8}$ extensively because sulfides, selenides, and tellurides are present as nonmetallic phases in steels. The compounds $\mathrm{MnS}$, $\mathrm{MnSe}$, and the high-temperature form of MnTe possess the $\mathrm{NaCl}$ type structure, leading to easy deformation of the manganese compounds, particularly at high temperatures. ${ }^{9}$ In contrast, the corresponding binary iron compounds (and the low-temperature form of $\mathrm{MnTe}$ ) are hexagonal and less readily deformed.

Manganese chalcogenides dissolve significant amounts of iron into solid solution by cation substitution. ${ }^{3-5}$ Previous studies of anion substitution reveal complete solubility between $\mathrm{MnS}$ and $\mathrm{MnSe}^{10}$ and between $\mathrm{MnSe}$ and the cubic form of $\mathrm{MnTe},{ }^{11}$ very limited solubility $(\approx 1 \%)$ between $\mathrm{MnS}$ and $\mathrm{MnO},{ }^{12}$ and moderate solubility $(8 \%)$ between $\mathrm{MnS}$ and cubic MnTe. ${ }^{13}$

There is interest in the phase relations between manganese and calcium chalcogenides because Chao and Van Vlack ${ }^{14}$ reported that

Presented at the 80th Annual Meeting. The American Ceramic Society, Detroit, Michigan, May 8. 1978 (Basic Science Division No. 7-B-78). Received May 15 1978: revised copy received December $26,1978$.

Based on a thesis submitted by Chi-Hung Leung for the Ph.D. degree in Metallurgical Engineering. The University of Michigan. Ann Arbor. Michigan.

Supported by the Materials Science and Engineering Fund, The University of Michigan.

${ }^{\star}$ Member, the American Ceramic Society. a few percent of $\mathrm{CaS}$ in solution hardens $\mathrm{MnS}$. This reaction has technological significance because calcium has been used to obtain "shape control" of MnS phases in rolled steel. ${ }^{15}$ Presumably this microstructural effect occurs because of solution hardening. Therefore, the extent of mutual solubility is desirable information. ${ }^{7,16-18}$ The present paper focuses on solubility limits in the systems $\mathrm{Ca}(\mathrm{VI})-\mathrm{Mn}(\mathrm{VI})$.

Attention to the structural variations of solid solutions dates back more than 50 years, when Vegard ${ }^{19-21}$ studied lattice changes within mixed crystals, i.e. solid-solution compounds. Specific attention was directed to the $\mathrm{NaCl}$-type compounds by Havighurst $e t$ $a l .{ }^{22}$ They used the differences in lattice parameters and found that, if two salts with the same structure had lattice constants of $a_{1}$ and $a_{2}$, and if

$$
\delta=\left\{\left(a_{1}-a_{2}\right) /\left[\left(a_{1}+a_{2}\right) / 2\right]\right\} \leq 0.05
$$

solid solutions could form. In the context of the present study, this result indicates that twice the difference in two cation sizes must be $<5 \%$ of the average lattice constant, since we are considering the $\mathrm{NaCl}$-type structure. However, their studies were limited to alkali halides which have predominantly ionic bonds. Work by Primak et $a l .^{23}$ on MnS-SrS and CaS-MgS showed greater solubility. Kondrashev and Omelchenko, ${ }^{24}$ who studied the systems $\mathrm{ZnS}-\mathrm{MgS}$ and $\mathrm{ZnS}-\mathrm{CaS}$, concluded that the controlling effect of solid solubility was that of chemical bond type and not of radii of atoms or ions.

\section{Experimental Procedures}

\section{(1) Materials Preparation}

(A) Manganese Sulfide: $\mathrm{MnS}$ was prepared by reducing $\mathrm{MnSO}_{4}$ with $\mathrm{H}_{2} \mathrm{~S}$ gas at $900^{\circ} \mathrm{C}$ :

$$
3 \mathrm{MnSO}_{4}+4 \mathrm{H}_{2} \mathrm{~S} \stackrel{900^{\circ} \mathrm{C}}{\longrightarrow} 3 \mathrm{MnS}+4 \mathrm{SO}_{2}+4 \mathrm{H}_{2} \mathrm{O}
$$

According to Chao et al., ${ }^{2}$ it is also possible to reduce $\mathrm{MnSO}_{4}$ by sulfur vapor.

(B) Manganese Selenide and Manganese Telluride: MnSe and $\mathrm{MnTe}$ were prepared by reacting appropriate mixtures of the elements in a vacuum-sealed borosilicate glass* tube, following the procedure established by Riewald and Van Vlack. ${ }^{25,26}$

The MnS, MnSe, and MnTe were further purified by melting in a carbon crucible within a vacuum induction furnace. Microscopic

\footnotetext{
*Pyrex, Corning Glass Works, Coming, N.Y
} 
examination of the purified compounds revealed no oxide or other extraneous phases.

(C) Calcium Sulfide: $\mathrm{CaS}$ was obtained by reducing $\mathrm{CaO}$ with $\mathrm{H}_{2} \mathrm{~S}$ at $1000^{\circ} \mathrm{C}$ :

$$
\mathrm{CaO}+\mathrm{H}_{2} \mathrm{~S} \stackrel{1000^{\circ} \mathrm{C}}{\longrightarrow} \mathrm{CaS}=\mathrm{H}_{2} \mathrm{O}
$$

(D) Calcium Selenide and Calcium Telluride: CaSe and $\mathrm{CaTe}$ were prepared from the elements; however, extreme care was required since the reaction is sufficiently exothermic to easily become autocatalytic and to build up temporarily high vapor pressures. This problem was avoided by slow heating and by the presence of a temperature gradient within the borosilicate glass tube. Since metallic calcium accumulates an oxidized surface when stored in the laboratory, all calcium used was cut from the interior of bulk material immediately prior to sealing in an evacuated tube with the Se or Te. Under these conditions, the calcium surface remained bright. Furthermore, there was neither microscopic nor diffraction evidence of $\mathrm{CaO}$ as a separate phase in the final product. Lattice parameters for $\mathrm{CaSe}$ and $\mathrm{CaTe}$ are 5.932 and $6.363 \AA$, respectively. (These parameters compare with 5.924 and $6.356 \AA$ reported in $\mathrm{X}$-ray diffraction tables. ${ }^{27}$ ) As with the manganese compounds studied by Chao et $a{ }^{12}{ }^{12}$ and Riewald and Van Vlack, ${ }^{25.26}$ the soluble oxygen levels in $\mathrm{CaS}, \mathrm{CaSe}$, and $\mathrm{CaTe}$ are expected to be very low.

Binary mixtures were prepared by grinding the selected compositions under acetone with an agate mortar and pestle. The mixtures were then pressed into $6-\mathrm{mm}$ pellets with a force of $\approx 4500 \mathrm{~N}(1000$ lb), giving a calculated pressure of $\approx 160 \mathrm{MPa}(23000 \mathrm{psi})$.

\section{(2) Equilibration}

Three procedures were used for thermal treatment, depending on the temperature. For equilibration at $\angle 1150^{\circ} \mathrm{C}$, the samples were sealed inside evacuated silica tubes. To avoid chemical reaction, the Te samples were isolated from the $\mathrm{SiO}_{2}$ tube by a graphite insert. The tubes were heated in a wound resistance furnace and, following equilibration, were quenched in ice water.

Since the MnTe-rich phase tends to dissociate to $\mathrm{Mn}+\mathrm{MnTe}_{2}$, manganese powder was also added to the opposite end of the sample tubes which contained that phase. The manganese powder was physically separated from the sample powder to prevent any possible contamination. This procedure satisfactorily avoided $\mathrm{MnTe}_{2}$ formation. presumably through an increased manganese vapor pressure.

Samples were equilibrated at $1150^{\circ}$ to $1400^{\circ} \mathrm{C}$ in an $\mathrm{SiC}$ resistance furnace within an atmosphere of copper-deoxidized, desiccated Ar. The specimens were placed in a 6-cavity graphite container $\left(2.5 \mathrm{~cm}\right.$ in diam.) as described by Leung. ${ }^{28}$

Samples were tested at $>1400^{\circ} \mathrm{C}$ by placing the graphite sample holder in an induction furnace, where it served as the susceptor. The purified $\mathrm{Ar}$ atmosphere and the quenching procedures were the same as for the samples in the intermediate temperature range. ${ }^{28}$ In each case, the gas train was isolated from the external atmosphere by the use of a slight positive pressure and the usual ground glass joint seals.

A noble-metal thermocouple, calibrated against a secondary standard thermocouple, was used at $\leq 1400^{\circ} \mathrm{C}$. Temperatures were measured in the high-temperature range with an optical pyrometer which was calibrated against the melting temperature of $\mathrm{MnS}$ $\left(1610^{\circ} \mathrm{C}\right)$. The power supply could be held constant to about $\pm 15^{\circ}$ within a $2-h$ period.

In the $1150^{\circ}$ to $1400^{\circ} \mathrm{C}$ furnace, rapid cooling was achieved by pulling the sample holder into the cold end of the furnace chamber into an Ar blast. In the induction furnace, the sample temperature decreased as soon as the power was turned off so that it was $<500^{\circ} \mathrm{C}$ in $\approx 1 \mathrm{~min}$; however, the specimens were not removed from the furnace until they were at ambient temperatures, to avoid surface oxidation.

\section{(3) Sample Analyses}

Phase compositions were determined by microprobe analyses. Calibrations followed the procedure of Mann, ${ }^{29}$ who obtained accuracies of $\pm 1 \mathrm{~mol} \%$. Lattice constants were determined by an $\mathrm{X}$-ray procedure using a Guinier-type camera with a $100-\mathrm{mm}$ film cylinder. Silicon powder provided an intemal standard. A computer

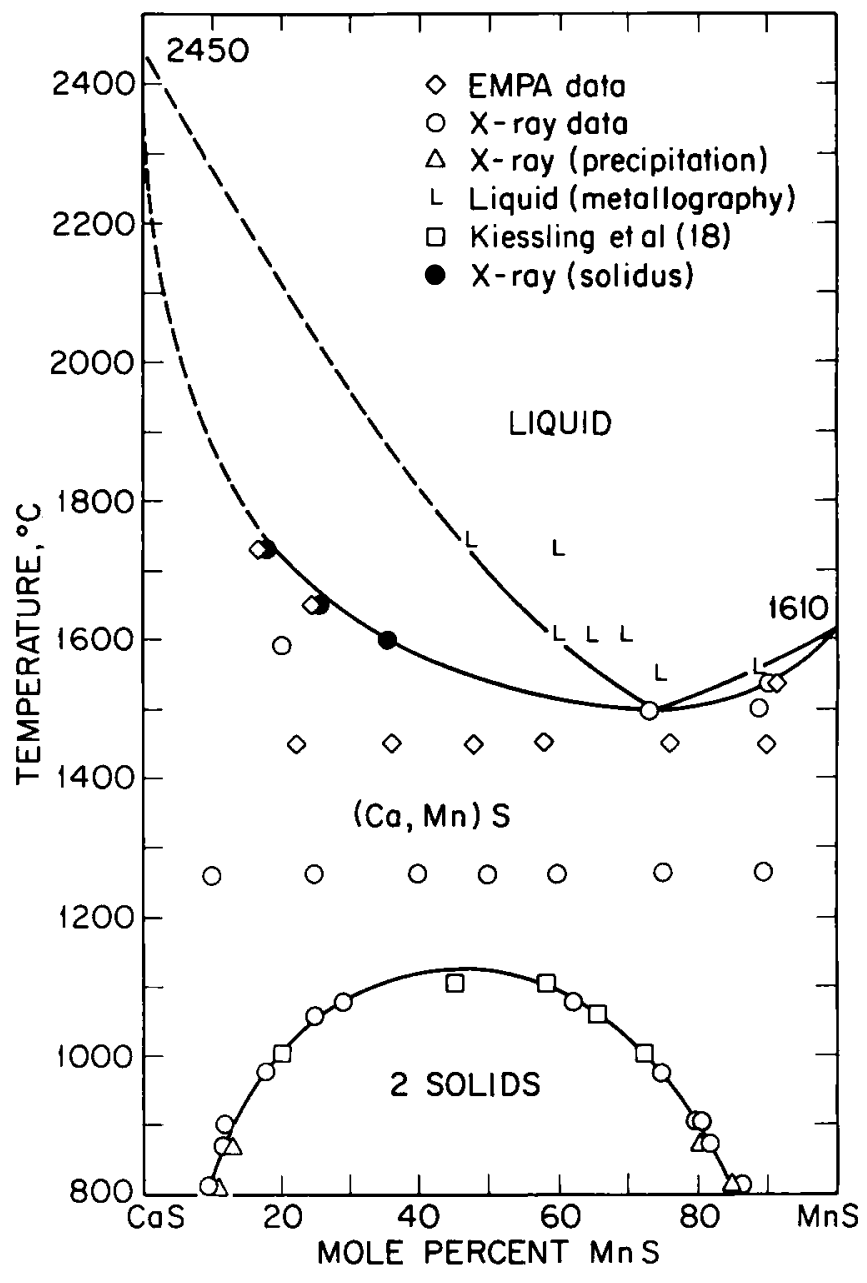

Fig. 1. The system CaS-MnS, drawn from results of this study, showing a miscibility gap and minimum melting point.

program with a LaGrange form of interpolation was used to calculate the lattice constants to $\pm 0.002 \AA .{ }^{30}$ This variance corresponds to about $\pm 0.5 \mathrm{~mol} \%$ difference in solid solubility for all systems studied. Details of the procedure and calculations involving the silicon internal standard are discussed elsewhere. ${ }^{28}$

The solvus and solidus boundaries within all three systems were determined by using data obtained from the preceding analyses. Liquid-containing regions of the phase diagrams were identified by microscopic examination.

The quenching procedures were rapid enough to avoid any evidence of subsolidus phase separation. Nor was there microscopic or $\mathrm{X}$-ray evidence that proeutectic solids were altered by the solidifying liquid during quenching from above the solidus.

\section{Results}

\section{(1) The System CaS-MnS}

The results of this study are shown in Fig. 1. Both $\mathrm{CaS}$ and MnS possess the $\mathrm{NaCl}$-type structure. Increased mutual solubility leads to full miscibility at $1150^{\circ} \mathrm{C}$. The solvus curve is slightly skewed in the CaS direction. The two points of data from the study by Kiessling and Westman ${ }^{7}$ fall on the more complete solvus of our figure.

Complete miscibility exists between $1150^{\circ}$ and $1500^{\circ} \mathrm{C}$, where a minimum occurs in the liquidus at $26 \mathrm{CaS}$ and $74 \mathrm{MnS}(\mathrm{mol} \%)$. Higher-temperature data extend the solidus to $1730^{\circ} \mathrm{C}$ at $15 \% \mathrm{MnS}$ and extrapolate the phase diagram to $2450^{\circ} \mathrm{C}$, the melting temperature of CaS, according to Vogel and Heumann. ${ }^{31}$ 


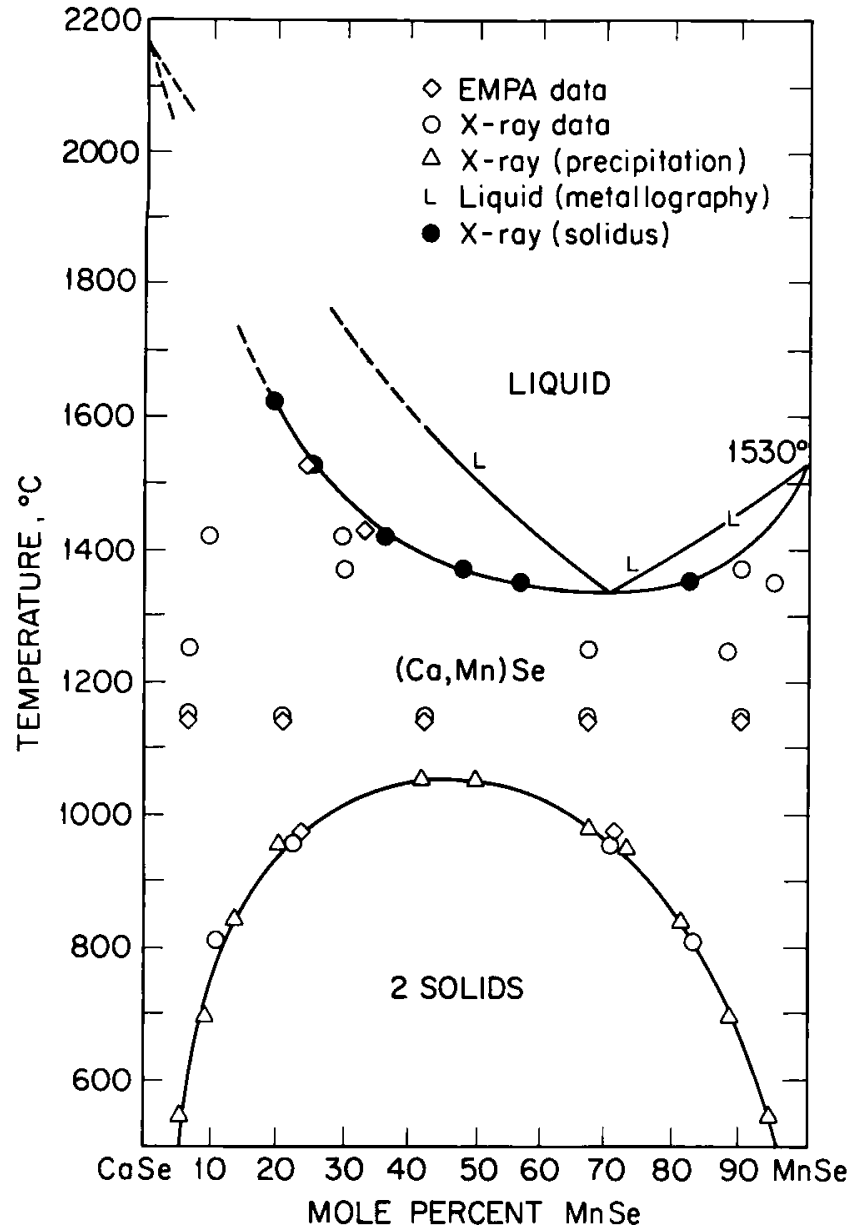

Fig. 2. The system CaSe-MnSe, drawn from results of this study, showing a miscibility gap and minimum melting point.

\section{(2) The System CaSe-MnSe}

This system parallels the system $\mathrm{CaS}-\mathrm{MnS}$ very closely, but at lower temperatures. The maximum of the miscibility gap is at $1070^{\circ} \mathrm{C}$, some $80^{\circ}$ below that in the sulfide. The liquidus minimum, which is $160^{\circ}$ below the minimum in the sulfide system, is at $1340^{\circ} \mathrm{C}$ and $30 \mathrm{CaSe}-70 \mathrm{MnSe}$. The melting temperature for $\mathrm{CaSe}$ is not known; however, an extrapolation of the solidus curve suggests a value $>2000^{\circ}$, but $<2450^{\circ} \mathrm{C}$, as reported for $\mathrm{CaS}{ }^{31}$

As with the sulfides, a slightly greater solid solubility occurs on the MnSe side of the miscibility gap than on the CaSe side. The phase diagram is shown in Fig. 2.

\section{(3) The System CaTe-MnTe}

The downward trend in liquidus temperatures from the sulfides to the selenides is continued to the tellurides (Fig. 3) with the result that the liquidus intercepts the miscibility gap to form a eutectic at $1050^{\circ} \pm 20^{\circ} \mathrm{C}(55 \mathrm{CaTe}-45 \mathrm{MnTe})$. Again, as with the sulfides and the selenides, the miscibility gap between the isomorphic phases is asymmetrically located nearer the CaTe side.

The system CaTe-MnTe is complicated by the polymorphic transition of $\mathrm{MnTe}$ from the $\mathrm{NaCl}$ structure (at $>1040^{\circ} \mathrm{C}$ ) to the $\mathrm{NiAs}$ structure below that temperature. This transition leads to a eutectoid reaction at $800^{\circ} \mathrm{C}$ and $10 \mathrm{CaTe}-90 \mathrm{MnTe}$. The maximum solubility of CaTe in the hexagonal polymorph is limited to $\approx 5 \mathrm{~mol} \%$.

\section{(4) Vegard's Law}

Figure 4 shows that the cation substitution produces an ideal change in the lattice parameter in the sulfide and telluride systems, since Vegard's law is followed linearly in those samples quenched from high temperature. There is a very slight, but detectable, positive variation from ideality in the selenide system.

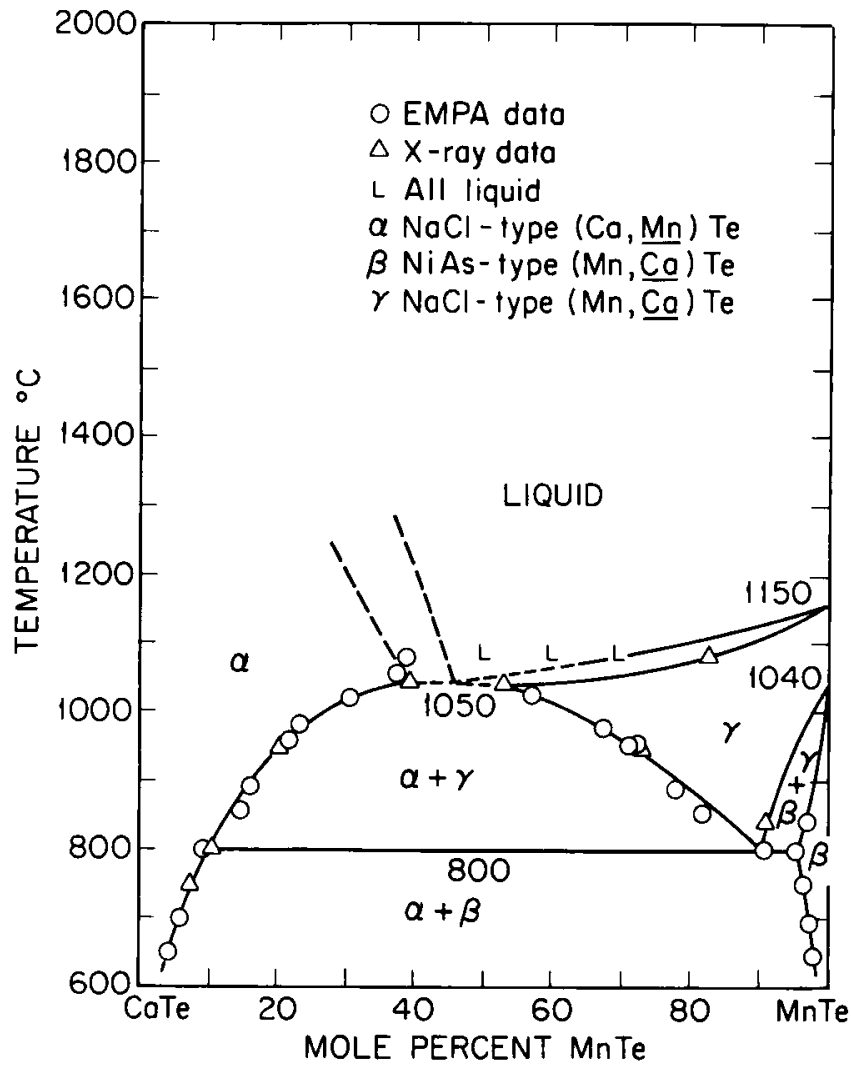

Fig. 3. The system CaTe-MnTe, drawn from results of this study, showing a eutectoid and eutectic point.

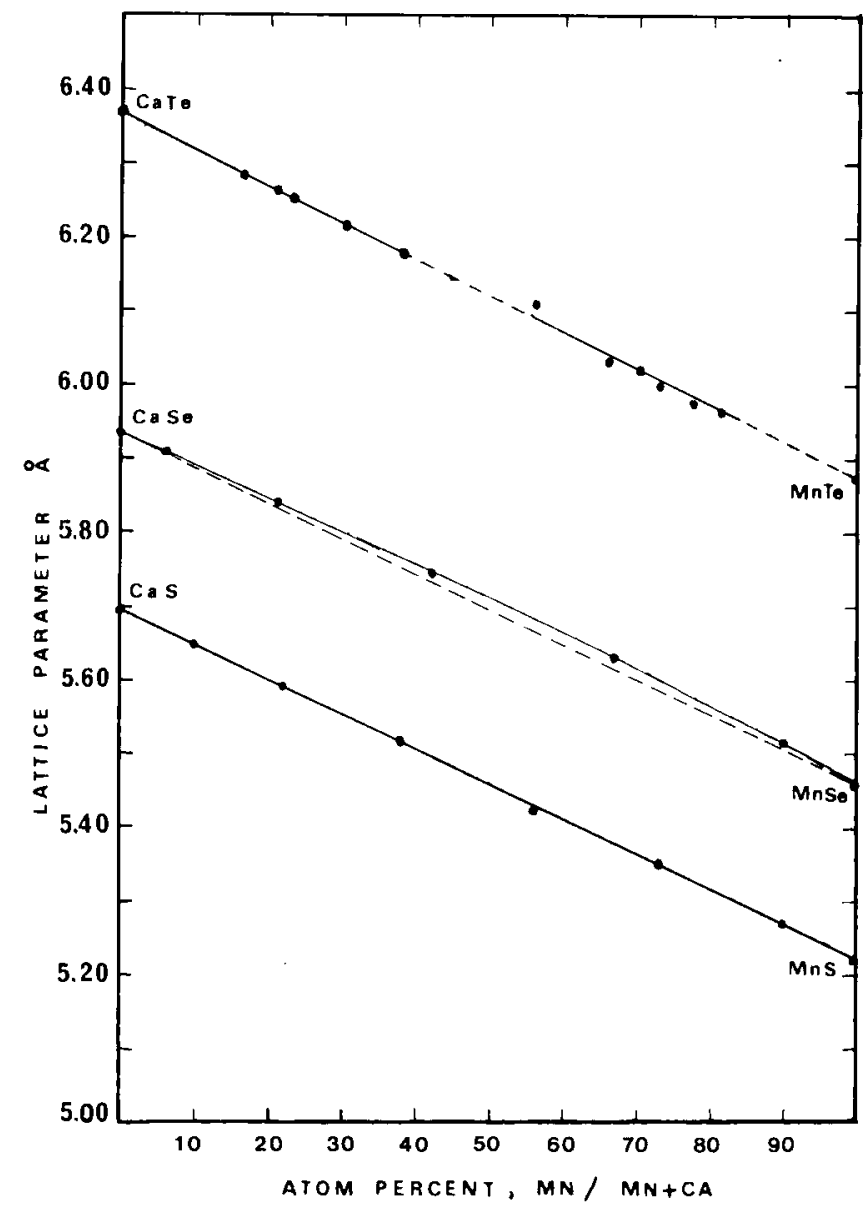

Fig. 4. Lattice parameters for cubic Ca-Mn chalcogenides. $\mathrm{CaTe}=6.363$, $\mathrm{CaSc}=5.932, \quad \mathrm{CaS}=5.694$, and $\mathrm{MnTe} \approx 5.875, \quad \mathrm{MnSe}=5.463$ $\mathrm{MnS}=5.224 \AA$. 


\section{Discussion}

The three binary chalcogenide systems, CaS-MnS, CaSe-MnSe, and CaTe-MnTe, may be compared directly. Except for the lowtemperature form of $\mathrm{MnTe}$, all solid phases are cubic $\mathrm{NaCl}$. Each system possesses a solid miscibility gap; each gap is slightly asymmetric to the $\mathrm{Ca}(\mathrm{VI})$ side. Each system has a low-melting liquid with the minimum toward the $\mathrm{Mn}$ (VI) side. The melting temperatures drop sequentially from the sulfides to the selenides to the tellurides (and, initially, from the oxides). Likewise, the solvus temperature decreases, but less drastically than the liquidus and solidus temperatures. The solid solutions in all three systems are nearly ideal as measured by Vegard's law.

The higher melting temperatures for the $\mathrm{Ca}(\mathrm{VI})$ than for the corresponding $\mathrm{Mn}$ (VI) compounds may be attributed to the greater ionicity of the $\mathrm{Ca}^{2+}$ ion as compared to that of $\mathrm{Mn}^{2+}$. In the presence of nearly ideal solid solutions, this melting differential would lead to the asymmetrical solidus curve which was observed. The progressive decrease in melting temperatures from sulfides to selenides to tellurides is consistent with that of other binary compounds, such as the alkali halides in downward sequences through the periodic table. Although this decrease may also be attributed to changes in ionicity from $S$ to $S e$ to $T e$, it is probably simpler to assign this temperature trend to the increase in the atom size and, therefore, reduced coulombic attractions.

In each of the $\mathrm{S}, \mathrm{Se}$, and Te series, the lattice constant for the $\mathrm{Ca}(\mathrm{VI})$ exceeds that of $\mathrm{Mn}(\mathrm{VI})$ by $\approx 0.475 \AA$, indicating that the radius of $\mathrm{Ca}^{2+}$ is consistently $0.24 \AA$ greater than that of $\mathrm{Mn}^{2+}$, independent of the anion when the anions are $S, S e$, and Te. This difference varies from $4.3 \%$ of the average sulfide lattice constant to $4.0 \%$ of that for the cubic telluride. This consistency probably accounts for the similarity of the solvus curves for the NaCl-type phases in each of the three systems.*

The very slight departure from Vegard's law for the selenides has no apparent explanation. Rather, it is surprising that subsolidus miscibility develops, in view of the near ideality found in all three systems. This reaction suggests that the miscibility gap arises solely from the difference in the cation size and not from Ca-Mn interactions.

\section{Conclusions}

Solid solutions within the three chalcogenide $\mathrm{Ca}-\mathrm{Mn}$ systems are nearly ideal with respect to Vegard's law. The miscibility gaps in the three systems permit less substitution to $\mathrm{Mn}^{2+}$ for $\mathrm{Ca}^{2+}$ than vice versa.

There is a systematic decrease in the liquidus temperatures from the sulfide to the selenide to the telluride systems. The solid misci-

\footnotetext{
*This consistency also suggests a possible miscibility gap in the system $\mathrm{CaO}-\mathrm{MnO}$ This system was not included in the present study of the Ca-Mn chalcogenides. Glasser (Ref. 32) said, "No evidence was obtained that a miscibility gap exists." It should be noted, however, that comparable systems once considered to be fully soluble have noted, however, that comparable systems once considered to be fully soluble have
subsequently been shown to have a miscibility gap, e.g. NiO-CoO (Ref. 33) and subsequently been show
}

bility gap also decreases, but less drastically; therefore, the liquidus and solvus intercept in the telluride system to produce a eutectic.

\section{References}

'H. Wentrup, "Oxide and Sulfide Enclosures in Steel," Tech. Mitr. Krupp, 5, $131-52(1937)$

${ }^{2}$ H. C. Chao, L. H. Van Vlack, F. Oberin, and L. Thomassen, "Hardness of Inclusion Sulfides," ASM Trans. $Q$, , 57 [4] 885-9] (1964).

${ }^{3}$ G. S. Mann and L. H. Van Vlack. "FeS-MnS Phase Relationships in the Presence of Excess Iron," Metall. Trans. B, 7B [3] 469-75 (1976).

'G. S. Mann and L. H. Van Vlack, "FeSe-MnSe Phase Relationships in the Presence of Excess Iron," ibid., 8B [1] 47-51 (1977).

${ }^{5} \mathrm{G}$. S. Mann and L. H. Van Vlack, "Fe ${ }_{1,2} \mathrm{Te}$-MnTe Phase Relationships in the Presence of Excess Iron," ibid., pp. 53-57.

${ }^{6} \mathrm{R}$. Kiessling and N. Lange, Nonmetallic Inclusions in Steel. The Metals Society (Iron and Steel Institute), London, 1966

${ }^{7}$ R. Kiessling and C. Westman, "Sulfide Inclusions and Synthetic Sulfides of the (Mn,Me)S-Type," J. Iron Steel Inst.. London, 204 [4] 377-79 (1966).

${ }^{B}$ R. Kiessling, B Hassler, and C. Westman, "Selenide-Sulfide Inclusions and Synthetic Compounds of the (Mn,Me)(S,Se)-Type," ibid., 205 [5] 531-34 (1967).

${ }_{9}^{9}$ H. C. Chao, "Deformation of Oriented Inclusions Within Steel"; Uni'. Micro/ilm Int. (Ann Arbor, Mich.) Order No. 65-5282, 107 pp.; Diss. Abstr., 25 [12] 7178 $(1965)$

${ }^{10} \mathrm{~J}$. M. Mehta, P. G. Riewald, and L. H. Van Vlack, "The System MnSe-MnS," $J$. Am. Ceram. Soc., 50 [3] 164 (1967).

${ }^{13}$ A. J. Panson and W. D. Johnson, "The MnTe-MnSe System," J Inorg. Nucl. Chem., 25 [5] 701-703 (1964)

${ }^{12}$ H. C. Chao, Y. E. Smith, and L. H. Van Vlack, "The MnO-MnS Phase Diagram," Trans. AIME, 227, 796-97 (1963).

${ }^{13}$ T. Y. Tien, R. J. Martin, and L. H. V an Vlack, "The MnTe-MnS System," ibid., 242 [10] 2153-54 (1968)

${ }^{1+}$ H. C. Chao and L. H. Van Vlack, "Deformation of Oriented MnS Inclusions in Low-Carbon Steel," ibid., 233 [7] 1227-31 (1965).

${ }^{15} \mathrm{~V}$. A. Tipnis, R. A. Joseph, and J. H. Doubrava "Improved Machining Steels Through Calcium Deoxidation," Metall. Eng. A, 13 [4] 39-47 (1973).

${ }^{16} \mathrm{E}$. A. Doroschkevich, R. Kiessling, and C. Westman, "The Solid Solubility of the Second Long-Period Transition Elements in $\alpha$-MnS,"J. Iron Steel Inst. London, 208 Second Long-Period
[Pt. 7] 698 (1970).

${ }^{17}$ D. C. Hilty and J. W. Farrell, "Modification of Inclusions by Calcium: II," /ron Steelmaker, 2 [6] 20-27 (1975).

${ }^{1 *} \mathrm{R}$. Kiessling and C. Westman, "The MnS-CaS System and Its Metallurgical Significance," J. Iron Steel Inst., London, 208 [Pt. 7] 699-700 (1970).

${ }^{19} \mathrm{~L}$. Vegard, "Formation of Mixed Crystals by Contact of Solid Phases and by Precipitation from Solutions." Z. P/ws., 42, 1-14 (1927).

${ }^{20} \mathrm{~L}$. Vegard, "Lattice Variations in the Formation of Mixed Crystals by Precipita${ }^{20} \mathrm{~L}$. Vegard, "Lattice Variations in the Form
tion of Solutions," ibid. , 43, 299-308 (1927).

${ }^{21}$ L. Vegard. "Constitution of Mixed Crystals and the Size of the Atom," ibid., 5, $17-26$ (1921)

${ }^{22}$ R. J. Havighurst, E. Mack, and F. C. Blake, "Solid Solution of the Alkali and Ammonium Halides," J. Am. Chem. Soc., 47, 29 (1925).

${ }^{23}$ W. Primak, H. Kaufman, and R. Ward, "X-Ray Diffraction Studies of Systems Involved in the Preparation of Alkaline Earth Sulfide and Selenide Phosphors," ibid.

70, 243-46 (1948)

${ }^{24}$ Yu. D. Kondrashev and Yu. A. Omelchenko, "X-Ray Study of Some Oxide and Sulfide Systems," Zh. Neorg. Khim., 9 [4] 937-43 (1964)

${ }^{2 s}$ P. G. Riewald and L. H. Van Vlack, "Slip Behavior and Hardness Indentations in MnSe and MnSe-MnS Solid Solutions," J. Am. Ceram. Soc., 52 [7] 370-75 (1969).

${ }^{26} \mathrm{P}$. G. Riewald and L. H. Van Vlack "Deformation and Fracture of $\mathrm{MnTe}$ and MnSe-MnTe Solid Solutions," ibid., 53 [4] 219-23 (1970).

${ }_{27}$ Powder Diffraction File, Card Nos. 18-304 and 15-880. Joint Committee on Powder Diffraction Standards. Swarthmore, Pa., 19081.

${ }^{2 *} \mathrm{C}$. H. Leung, "Solid Solutions and Hardnesses of Binary Compounds with in the (Ca,Mn)/(S,Se,Te) System"; Univ. Microfilms Int. (Ann Arbor, Mich.) Order No. 78-04750, 175 pp.; Diss. Absir. Im. B, 38 [11] 5524 (1978).

${ }^{29} \mathrm{G}$. S. Mann, "Phase Equilibria of Iron and Manganese with Sulfur, Selenium, and Tellurium", Univ. Microfilms Int. (Ann Arbor, Mich.) Order No. 75-10224, 144 pp.; Diss. Abstr. Int. B, 35 [1] 5455 (1975).

30 'Photography of Powder Diffraction Pattems with X-Ray Focussing Camera Model V-FXC." Incentive Research and Development AB Tech. Rept., Ranhammarsvagen, Bromma, Sweden.

${ }^{31}$ R. Vogel and T. Heumann, "The System Fe-FeS-CaS," Arch. Eisenhuttenn:, $15,195-99(1941)$

${ }^{32}$ F. P. Glasser, "The Temary Systems CaO-MnO-SiO ${ }_{2}$," J. Am. Ceram. Soc., 45 [5] 242-49 (1962)

${ }^{33} \mathrm{M}$. Kinoshita, W. D. Kingery, and H. K. Bowen, "Phase Separation in NiO-CoO Solid Solution Single Crystals," ibid., 56 [7] 398-99 (1973).

${ }^{3+}$ S. A. Chudinova, S. P. Kuz"kina, and O. N. Shivrin, "Kinetics of Ordering in an NiO-MgO Solid Solution," Sor. Phys.-Solid State, 11, 1217 (1970). 